\title{
Pain Related to External Injury and Disorders Associated with Psychosocial Factors
}

\author{
Kanaka Yatabe*1, Ryota Muroi ${ }^{1}$, Takanori Kumai ${ }^{1}$, Takashi Kotani ${ }^{2}$, Toshio Kumai ${ }^{3}$ and Hiroto Fujiya ${ }^{1}$ \\ ${ }^{1}$ Department of Sports Medicine, St. Marianna University School of Medicine, Japan \\ ${ }^{2}$ Department of Orthopaedic Surgery, St. Marianna University School of Medicine, Japan
}

${ }^{3}$ Department of Pharmacogenomics, St. Marianna University Graduate School of Medicine, Japan

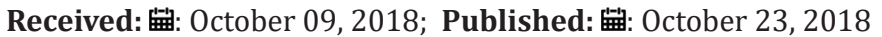

*Corresponding author: Kanaka Yatabe, Department of Sports Medicine, St. Marianna University School of Medicine, Japan

\section{Introduction}

In many sports, there are cases in which psychosocial factors are involved in the onset and persistence of lower back and lower limb pain. For example, the diagnostic guidelines for chronic pain and lumbar disc herniation state that psychological disorders are associated with the prognosis of these conditions [1]. Onset of chronic pain is especially difficult to manage as various stress factors, in addition to the physiological factors, complicate the condition. Various reports have described anxiety or depression, antisocial behavior, and social anxiety disorders in animal models, and a novel report has suggested the possibility of psychological factors intensifying radicular pain arising from intervertebral disc herniation [2-5].

However, in actual sporting activities, it is difficult to assess whether psychological factors are the cause of pain or if the experience of pain leads to the development of anxiety and depression. Pain like this consists of both sensory and emotional aspects, as defined by the International Association for the Study of Pain [1]. This resembles the emotional mechanism of whether one cries because one is sad or whether one becomes sad because one cries. While in some cases psychological conditions improve in tandem with postoperative recovery, some athletes show little improvement in psychological condition.

\section{Background \& Review of Literature}

There are two types of pain that should be understood: acute pain and chronic pain. Acute pain has a specific physiological cause such as an external injury or inflammation, the site and degree of which can be easily determined. Chronic pain, by contrast, is defined as any pain that persists for longer than the normal recovery process for an acute condition or the reasonable time for a wound to heal [6] and is regarded as prolonged recursive pain. The pain is not localized but found across the body and it is difficult to specify the site or exact cause or to provide a medical explanation. Chronic pain also negatively impacts homeostasis and disrupts the balance of the autonomic nervous system, endocrine system and immune system. Therefore, malaise such as insomnia also increases, making it difficult to handle the pain. In sports, prolonged and unmanageable pain can be a stressor that adversely impacts athletes both psychologically and physiologically, often leading to the onset of chronic disorders, creating a negative cycle of pain and accumulation of stress (Table 1).

Table 1: Biological pathways, candidate gene with the pain related with QOL.

\begin{tabular}{|c|c|}
\hline Biological pathways & Candidate gene \\
\hline \multirow{4}{*}{$\begin{array}{l}\text { Cytokine-cytokine receptor interaction } \\
\text { Pro-inflammatory }\end{array}$} & $I L-1 \beta$ \\
\hline & $I L-6$ \\
\hline & $I L-8$ \\
\hline & $T N F-\alpha$ \\
\hline \multirow{3}{*}{ Anti-inflammatory } & $I L-1 R N$ \\
\hline & $I L-1 R A$ \\
\hline & $I L-10$ \\
\hline \multirow{2}{*}{ Dopaminergic synapse } & COMT \\
\hline & CREB1 \\
\hline \multirow{2}{*}{ Serotonergic synapse } & 5-HTT (SLC6A4) \\
\hline & TPH1 \\
\hline Neuroactive ligand-receptor interaction & OPRM1 \\
\hline
\end{tabular}

Injury or disorder-related pain is often seen in sports such as American football, figure skating and basketball, and during 
our several years of research focusing on ballet dancers' injuries, we found that compared to the observation period in which no intervention occurred, the rate of external injury or disorder gradually decreased during the period of biweekly medical check and interview and the period in which additional support such as treatment was provided as follow-up. Lower rates of external injury or disorder and stabilization of the subjects' moods were achieved by enhancing and continuously providing medical support [7]. During the long-term intervention period, Serotonin Transporter Gene Polymorphism (5-HTTLPR) was also found to have a main and interaction effect on mood [8]. In addition, there is the possibility that the psychological conditions related to personality traits and emotions are also affected at the level of gene polymorphism. Currently, we are conducting an additional investigation of its potential correlation with Catechol-O-Methyltransferase (COMT).

Based on the results of genetic analysis of the biological pathways involved in Quality Of Life (QOL) in Table 1, such as pain, fatigue, depression, and other emotional and social disorders, genes such as COMT, involved in the inactivation of cytokine and catecholamines (dopamine, adrenaline, and noradrenaline); serotonin transporter, related to Serotonin (5-HTT) and Tryptophan Hydroxylase 1 (TPH1) can be predicted to play a role in psychological disorders like pain and depression $[9,10]$. Chronic pain, in particular, is a pathological response to stress, as it is common to observe correlations with psychological and physiological conditions, chronic pain may be considered a typical example of psychosomatic illness.

\section{Conclusion}

In sports, pain symptoms are thought to be impacted primarily by social problems such as anxiety, depression, anger, nervousness, and frustration, and may greatly influence one's career. The articles were announced about the relationship between genotypes such as COMT or 5-HTTLPR and concussion history in rugby players [11]. It greatly affects daily life thought to be their physical impediment, that is to say QOL. We can find more biomedical research concerning the effects of the cytokine, dopamine and serotonin synapses on pain and psychological stress symptoms is expected to be conducted in the future, regardless of the field of sport.

\section{References}

1. International association for the study of pain (1979) Pain terms: A list with definitions and notes on usage. Recommended by the IASP Subcommittee on Taxonomy. Pain 6(3): 249-252.

2. Kumar V, Bhat ZA, Kumar D (2013) Animal modes of anxiety: A comprehensive review. J Pharmacol Toxicol Methods 68: 175-183.

3. Toth I, Neumann ID (2013) Animal models of social avoidance and social fear. Cell Tissue Res 354: 107-118.

4. Uesugi K, Sekiguchi M, Kikuchi S, Konno S (2011) The effect of repeated restraint stress in pain-related behavior induced by nucleus pulposus applied on the nerve root in rats. Eur Spine J 20: 1885-1891.

5. Fonseka TM, Wen XY, Foster JA, Kennedy SH(2016) Zebrafish models of major depressive disorders. J Neurosci Res 94(1): 3-14.

6. American society of anesthesiologists task force on chronic pain management (2010) Practice guidelines for chronic pain management. Anesthesiology 112: 810-833.

7. Yatabe K, Yui N, Kasuya S, Fujiya H, Tateishi K, et al. (2014) Anxiety and mood among Ballet dancers: A pilot study on effects of a medical approach involving periodic intervention. Ann Sports Med Res 1: 1002.

8. Yatabe K, Kumai T, Fujiya H, Yui N, Kasuya S, et al. (2016) Effects of serotonin transporter gene polymorphism on mood during the period before the competition in Japanese ballet dancers. Integr Mol Med 3: 818-825.

9. Spranger MA, Sloan JA, Barsevick A, Chauhan C, Dueck AC, et al. (2010) Scientific imperatives, clinical implications, and theoretical underpinnings for the investigation of the relationship between genetic variables and patient-reported quality-of-life outcomes. Qual Life Res 19(10): 1395-1403.

10. Spranger MA, Thong MS, Bartels M, Barsevick A, Ordoñana J, et al. (2014) Biological pathways, candidate genes, and molecular markers associated with quality-of-life domains: An update. Qual Life Res 23(7): 1997-2013.

11. Mc Fie S, Abrahams S, Patricios J, Suter J, Posthumus M, et al. (2018) The association between COMT rs4680 and 5-HTTLPR genotypes and concussion history in South African rugby union players. J Sports Sci 36(8): 920-933.

\section{ISSN: 2574-1241}

DOI: 10.26717/BJSTR.2018.10.001924

Kanaka Yatabe. Biomed J Sci \& Tech Res

CC (9) This work is licensed under Creative Commons Attribution 4.0 License

Submission Link: https://biomedres.us/submit-manuscript.php

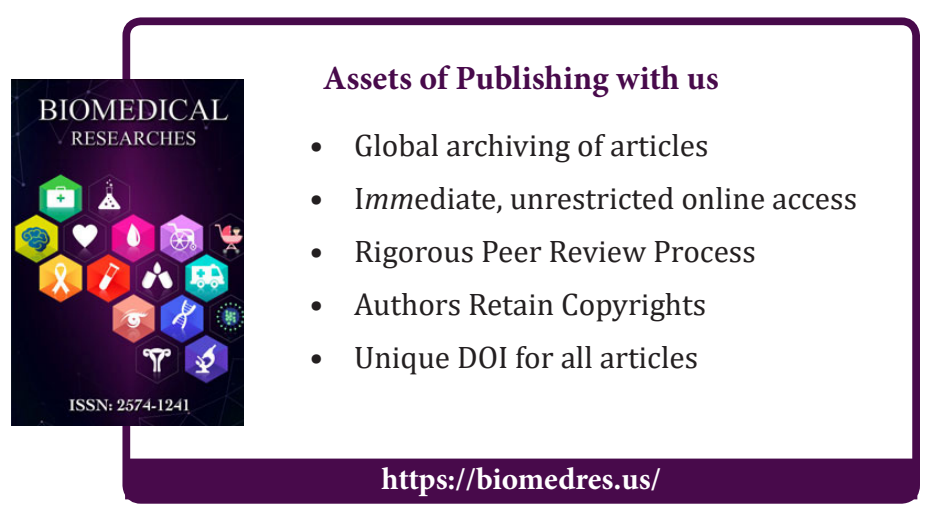

Cite this article: Kanaka Y, Ryota M, Takanori K, Takashi K, Toshio K, Hiroto F. Pain Related to External Injury and Disorders Associated with 